\title{
A light scattering model for total internal reflection microscopy of geometrically anisotropic particles
}

\author{
Adrian Doicu ${ }^{1 *}$ Alina A. Vasilyeva ${ }^{2}$, Dmitry S. Efremenko ${ }^{1}$, \\ Christopher L. Wirth ${ }^{3}$, Thomas Wriedt ${ }^{4}$
}

\author{
${ }^{1}$ Deutsches Zentrums fuer Luft- und Raumfahrt (DLR), Institut of Remote Sensing, Oberpfaffenhofen, Germany \\ ${ }^{2}$ University of Applied Sciences Rosenheim, Faculty of Electrical Engineering and Information Technology, \\ Rosenheim, Germany \\ ${ }^{3}$ Chemical and Biomedical Engineering Department, Cleveland State University, Cleveland, Ohio, USA \\ ${ }^{4}$ Leibniz-Institut fuer Werkstofforientierte Technologien - IWT, University of Bremen, Bremen, Germany
}

\begin{abstract}
In this paper a light scattering model for Total Internal Reflection Microscopy (TIRM) is described. The model handles the scattering by an axisymmetric particle of arbitrary orientation situated in the evanescent field near a plane surface, and the imaging of the scattered light via microscope optics. The scattering problem is solved by using the T-matrix method and the rotation addition theorem for spherical vector wave functions, while the image of the scattered field is computed by using the Debye diffraction integral. The numerical simulations provide evidence of two working regimes for TIRM: the first regime, corresponding to an incident angle less than the critical angle of total internal reflection, gives information on the size and the orientation of the particle, while the second regime, corresponding to an incident angle larger than the critical angle of total internal reflection, is recommended for measuring the distance between the particle and plane surface.
\end{abstract}

Keywords — Total internal reflection microscopy; light scattering model; T-matrix

\section{Introduction}

Total Internal Reflection Microscopy (TIRM) has proven to be an effective technique to measure weak interaction forces between spherical colloidal particles and surfaces with a resolution of a few femtonewton [1]. In an experimental setup a laser beam is coupled into a prism and hits the glass-water interface with an angle slightly above the critical angle of total internal reflection. This generates an evanescent field near the interface that decays in the lower refractive index medium (water) with a characteristic penetration depth which depends on the angle of incidence. A colloidal particle that is dispersed in the medium will scatter light from the evanescent field if it is in the vicinity of the surface. TIRM has proven to be a valuable tool for the precise measurement of weak colloidal interactions such as double layer forces [2, 3], van der Waals forces [4, 5], electrically mediated interactions [6, 7], polymer mediated steric repulsion $[8,9]$, and depletion forces $[10,11]$. An exhaustive review on TIRM can be found in Ref. [12, 13].

To design a TIRM instrument for a specific measuring problem, a light scattering model is needed. Essentially, we have to model the scattering by an axisymmetric particle of arbitrary orientation situated near a plane surface, and the imaging of the scattered light through an optical system. Either a photomultiplier tube that automatically integrates the scattered light signal or a digital camera that collects an image of the scattering [13], which is then integrated, are used to conduct TIRM. As the scattering problem is a multiple scattering problem the separation of variables technique is a highly suitable simulation approach. The approach based on the T-Matrix method was originally developed by Helden et al. [14] and it has been validated by comparing to measurents [15].

\footnotetext{
*CONTACT Adrian Doicu. Email: adrian.doicu@dlr.de
} 
To model the scattering problem in the framework of the separation of variables technique one must address how the radiation interacts with the particle and with the plane interface. The incident field strikes the particle directly, while the fields emanating from the particle may reflect off the surface and interact with the particle again. The transition matrix relating the incident and scattered field coefficients is computed in the framework of the T-matrix method, and the reflection matrix characterizing the reflection of the scattered field by the surface is computed by using the integral representation for the spherical vector wave functions. To handle an arbitrary particle orientation we will use the rotation addition theorem for spherical vector wave functions. Essentially, this theorem will enable us to pass from the field expansions in the global coordinate system to the field expansions in the particle coordinate system and vice versa. In general, if the particle coordinate system $O_{\mathrm{o}} x_{\mathrm{p}} y_{\mathrm{p}} z_{\mathrm{p}}$ is obtained by rotating the global coordinate system $O_{\mathrm{o}} x_{\mathrm{o}} y_{\mathrm{o}} z_{\mathrm{o}}$ thought the Euler angles $\left(\alpha_{\mathrm{p}}, \beta_{\mathrm{p}}, \gamma_{\mathrm{p}}\right)$, the rotation addition theorem for spherical vector wave functions is [16]

$$
\begin{aligned}
& \mathbf{M}_{m n}^{1,3}(k r, \theta, \varphi)=\sum_{m^{\prime}=-n}^{n} D_{m m^{\prime}}^{n}\left(\alpha_{\mathrm{p}}, \beta_{\mathrm{p}}, \gamma_{\mathrm{p}}\right) \mathbf{M}_{m^{\prime} n}^{1,3}\left(k r, \theta_{\mathrm{p}}, \varphi_{\mathrm{p}}\right), \\
& \mathbf{N}_{m n}^{1,3}(k r, \theta, \varphi)=\sum_{m^{\prime}=-n}^{n} D_{m m^{\prime}}^{n}\left(\alpha_{\mathrm{p}}, \beta_{\mathrm{p}}, \gamma_{\mathrm{p}}\right) \mathbf{N}_{m^{\prime} n}^{1,3}\left(k r, \theta_{\mathrm{p}}, \varphi_{\mathrm{p}}\right),
\end{aligned}
$$

where $D_{m m^{\prime}}^{n}$ are the Wigner $D$-functions, $(r, \theta, \varphi)$ are the spherical coordinates of a field point in the global coordinate system, and $\left(r, \theta_{\mathrm{p}}, \varphi_{\mathrm{p}}\right)$ are the spherical coordinates of the same field point in the particle coordinate system. A direct consequence of the arbitrary particle orientation is that the scattering problem will not decouple over the azimuthal modes as it happens when the axis of symmetry of the particle is perpendicular to the plane surface [17].

As an imaging system we consider pairs of lenses arranged in a $4 \mathrm{f}$ configuration, that is to say they are placed such that their foci coincide, for both illumination and image formation. The particle is placed in the front focal plane of the first lens, such that a corresponding image field results in the back focal plane of the second lens in a phase correct manner. Critically, the aperture stop is placed in the common focal plane of the lens pair, yielding an afocal and telecentric system from both object and image spaces.

The next parts of the paper are organized as follows. In Section 2 we present the mathematical model of TIRM dealing with scattering by an axisymmetric particle of arbitrary orientation situated near a plane surface, followed by imaging of the scattered light. A numerical analysis of the TIRM model is the objective of Section 3, while conclusions are formulated in Section 4.

\section{Theory}

The geometry of the scattering problem and the imaging system are shown in Fig. 1. An axisymmetric particle of arbitrary orientation is situated in the neighborhood of a plane surface $\Sigma$. The imaging system is represented by the two sections of the Gaussian reference spheres of the collector and detector lens, $P_{\mathrm{o}}$ and $P_{\mathrm{i}}$, respectively. Let $O_{\mathrm{o}} x_{\mathrm{o}} y_{\mathrm{o}} z_{\mathrm{o}}$ be a coordinate system centered at the particle, and in this way at the focal point of the collector lens, and $O_{\mathrm{i}} x_{\mathrm{i}} y_{\mathrm{i}} z_{\mathrm{i}}$ a coordinate system centered at the focal point of the detector lens. In the (global) coordinate system $O_{\mathrm{o}} x_{\mathrm{o}} y_{\mathrm{o}} z_{\mathrm{o}}$, the axial position of the plane surface $\Sigma$, which is perpendicular to the $z_{\mathrm{o}}$-axis, is specified by the distance $z_{0}$, and we have $z_{0}=d+R$, where $R$ is the radius of the sphere circumscribing the particle and $d$ is the effective distance between the particle and the plane surface (i.e., the distance between the plane surface and the circumscribing sphere). Moreover, in the coordinate system $O_{\mathrm{o}} x_{\mathrm{o}} y_{\mathrm{o}} z_{\mathrm{o}}$, let the orientation of the particle be described by the Euler orientation angles $\left(\alpha_{\mathrm{p}}, \beta_{\mathrm{p}}\right)$. The refractive index of the particle is $n_{\mathrm{p}}$, the refractive index of the substrate is $n_{\mathrm{s}}$, and the refractive indices in the object space (ambient medium) and the image space are $n_{\mathrm{o}}$ and $n_{\mathrm{i}}$, respectively. The focal lengths of the collector and detector lens are $f_{\mathrm{o}}$ and $f_{\mathrm{i}}$, respectively.

\subsection{Scattering by the particle near the substrate}

The incident field is a linearly polarized vector plane wave propagating in the glass substrate and is given by

$$
\mathbf{E}_{0}(\mathbf{r})=\left(E_{0 \beta} \mathbf{e}_{\beta}+E_{0 \alpha} \mathbf{e}_{\alpha}\right) \mathrm{e}^{\mathrm{j} \mathbf{k}_{\mathbf{s}} \cdot \mathbf{r}} .
$$

The incident wave vector $\mathbf{k}_{\mathrm{s}}=k_{\mathrm{s}} \mathbf{e}_{k}$, where $k_{\mathrm{s}}=k_{0} n_{\mathrm{s}}$ is the wavenumber in the substrate, $k_{0}$ is the wavenumber in free space, and $\left(\mathbf{e}_{k}, \mathbf{e}_{\beta}, \mathbf{e}_{\alpha}\right)$ are the spherical unit vectors associated to $\mathbf{k}_{\mathrm{s}}$, is assumed to be in the $x_{\mathrm{o}} z_{\mathrm{o}}$-plane and to 


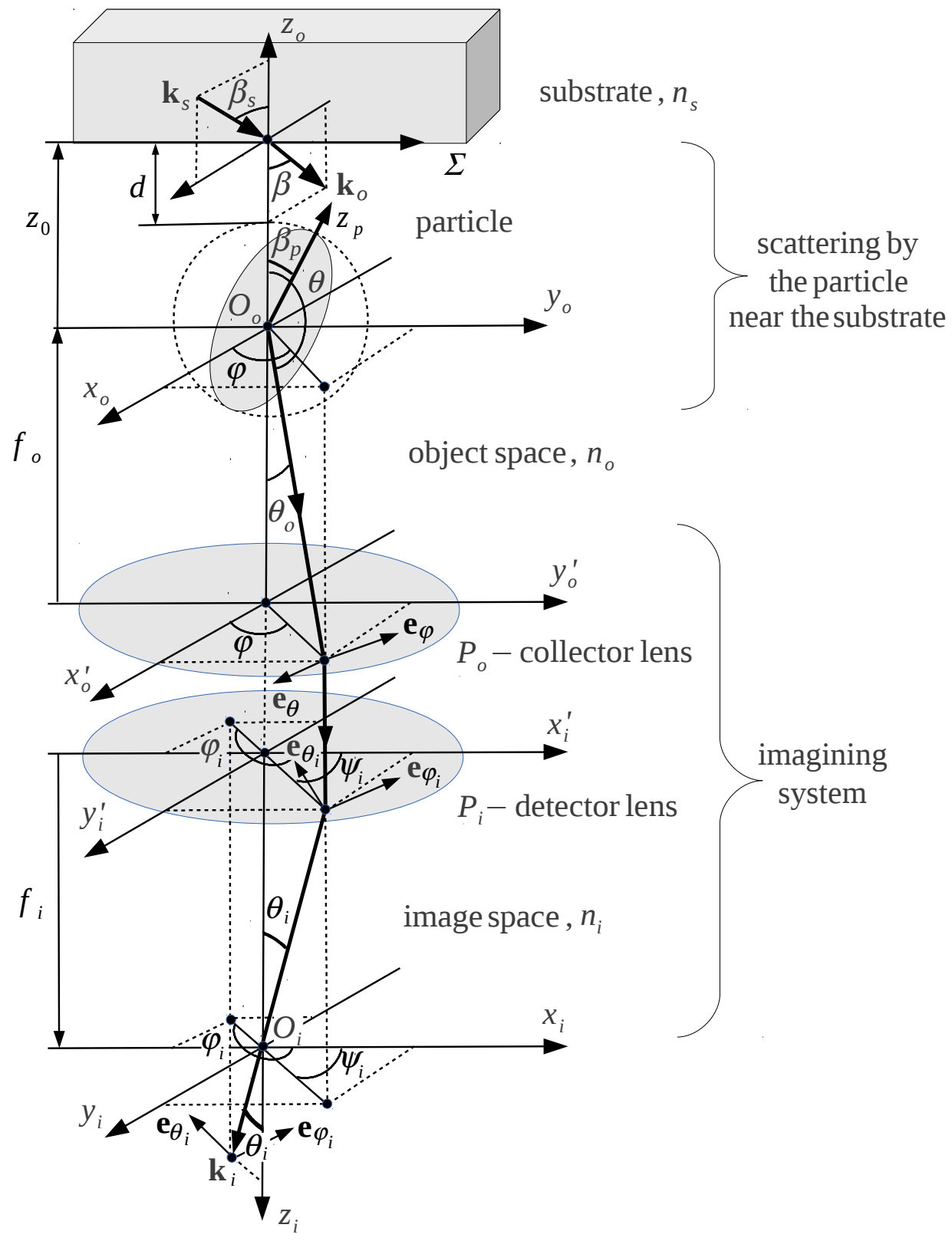

Figure 1: Geometry of the scattering problem and the imaging system. 
enclose the angle $\beta_{\mathrm{s}}$ with the $z_{\mathrm{o}}$-axis. The vector plane wave transmitted (or refracted) into the ambient medium is

$$
\mathbf{E}_{0}^{\mathrm{T}}(\mathbf{r})=\left(E_{0 \beta}^{\mathrm{T}} \mathbf{e}_{\beta \mathrm{T}}+E_{0 \alpha}^{\mathrm{T}} \mathbf{e}_{\alpha \mathrm{T}}\right) \mathrm{e}^{\mathrm{j} \mathbf{k}_{\circ} \cdot \mathbf{r}},
$$

where

$$
\begin{aligned}
& E_{0 \beta}^{\mathrm{T}}=t_{\|}\left(\beta_{\mathrm{s}}\right) \mathrm{e}^{\mathrm{j} k_{\mathrm{o}} z_{0}\left(\cos \beta-m_{\mathrm{rs}} \cos \beta_{\mathrm{s}}\right)} E_{0 \beta}, \\
& E_{0 \alpha}^{\mathrm{T}}=t_{\perp}\left(\beta_{\mathrm{s}}\right) \mathrm{e}^{\mathrm{j} k_{\mathrm{o}} z_{0}\left(\cos \beta-m_{\mathrm{rs}} \cos \beta_{\mathrm{s}}\right)} E_{0 \alpha},
\end{aligned}
$$

$\mathbf{k}_{\mathrm{o}}=k_{\mathrm{o}} \mathbf{e}_{k \mathrm{~T}}$ is the transmitted wave vector, $k_{\mathrm{o}}=k_{0} n_{\mathrm{o}}$ is the wavenumber in the ambient medium, $m_{\mathrm{rs}}=n_{\mathrm{s}} / n_{\mathrm{o}}$ is the relative refractive index of the substrate with respect to the ambient medium, and $\left(\mathbf{e}_{k \mathrm{~T}}, \mathbf{e}_{\beta \mathrm{T}}, \mathbf{e}_{\alpha \mathrm{T}}\right)$ are the spherical unit vectors associated to $\mathbf{k}_{\mathrm{o}}$. The Fresnel transmission coefficients are given by

$$
\begin{aligned}
t_{\|}\left(\beta_{\mathrm{s}}\right) & =\frac{2 m_{\mathrm{rs}} \cos \beta_{\mathrm{s}}}{\cos \beta_{\mathrm{s}}+m_{\mathrm{rs}} \cos \beta}, \\
t_{\perp}\left(\beta_{\mathrm{s}}\right) & =\frac{2 m_{\mathrm{rs}} \cos \beta_{\mathrm{s}}}{m_{\mathrm{rs}} \cos \beta_{\mathrm{s}}+\cos \beta},
\end{aligned}
$$

while the angle of refraction is computed by means of Snell's law:

$$
\begin{aligned}
& \sin \beta=m_{\mathrm{rs}} \sin \beta_{\mathrm{s}}, \\
& \cos \beta= \pm \sqrt{1-\sin ^{2} \beta .}
\end{aligned}
$$

Evanescent waves appear for real $m_{\mathrm{rs}}$ and incident angles $\beta_{\mathrm{s}}>\beta_{\mathrm{sc}}$, where $\beta_{\mathrm{sc}}=\arcsin \left(1 / m_{\mathrm{rs}}\right)$ is the angle of total internal reflection. In this case, $\sin \beta>1$ and $\cos \beta$ is purely imaginary. For $\mathbf{r}=\left(x_{\mathrm{o}}, y_{\mathrm{o}}, z_{\mathrm{o}}\right)$ and negative values of $z_{0}$, we have

$$
\exp \left(\mathrm{jk}_{\mathrm{o}} \cdot \mathbf{r}\right)=\exp \left(\mathrm{j} k_{\mathrm{o}}\left|z_{\mathrm{o}}\right| \cos \beta+\mathrm{j} k_{\mathrm{o}} x_{\mathrm{o}} \sin \beta\right),
$$

and we choose the sign of the square root such that $\operatorname{Im}(\cos \beta)>0$. This choice guarantees that the amplitude of the refracted wave propagating in the negative direction of the $z_{0}$-axis decreases with increasing the distance $\left|z_{\mathrm{o}}\right|$. In the global and particle coordinate systems, the transmitted incident fields can be expanded in terms of regular spherical vector wave functions, that is,

$$
\begin{aligned}
\mathbf{E}_{0}^{\mathrm{T}}(\mathbf{r}) & =\sum_{n=1}^{\infty} \sum_{m=-n}^{n} A_{m n}^{\mathrm{T}} \mathbf{M}_{m n}^{1}\left(k_{\mathrm{o}} r, \theta, \varphi\right)+B_{m n}^{\mathrm{T}} \mathbf{N}_{m n}^{1}\left(k_{\mathrm{o}} r, \theta, \varphi\right) \\
& =\sum_{n=1}^{\infty} \sum_{m=-n}^{n} a_{m n}^{\mathrm{T}} \mathbf{M}_{m n}^{1}\left(k_{\mathrm{o}} r, \theta_{\mathrm{p}}, \varphi_{\mathrm{p}}\right)+b_{m n}^{\mathrm{T}} \mathbf{N}_{m n}^{1}\left(k_{\mathrm{o}} r, \theta_{\mathrm{p}}, \varphi_{\mathrm{p}}\right),
\end{aligned}
$$

where $(r, \theta, \varphi)$ are the spherical coordinates of a field point in the global coordinate system $O_{\mathrm{o}} x_{\mathrm{o}} y_{\mathrm{o}} z_{\mathrm{o}},\left(r, \theta_{\mathrm{p}}, \varphi_{\mathrm{p}}\right)$ are the spherical coordinates of the same field point in the particle coordinate system $O_{\mathrm{o}} x_{\mathrm{p}} y_{\mathrm{p}} z_{\mathrm{p}}$,

$$
\begin{aligned}
& A_{m n}^{\mathrm{T}}=-\frac{4 \mathrm{j}^{n}}{\sqrt{2 n(n+1)}}\left[\mathrm{j} m \pi_{n}^{|m|}(\beta) E_{0 \beta}^{\mathrm{T}}+\tau_{n}^{|m|}(\beta) E_{0 \alpha}^{\mathrm{T}}\right], \\
& B_{m n}^{\mathrm{T}}=-\frac{4 \mathrm{j}^{n+1}}{\sqrt{2 n(n+1)}}\left[\tau_{n}^{|m|}(\beta) E_{0 \beta}^{\mathrm{T}}-\mathrm{j} m \pi_{n}^{|m|}(\beta) E_{0 \alpha}^{\mathrm{T}}\right],
\end{aligned}
$$

and

$$
\begin{aligned}
& a_{m^{\prime} n}^{\mathrm{T}}=\sum_{m=-n}^{n} A_{m n}^{\mathrm{T}} D_{m m^{\prime}}^{n}\left(\alpha_{\mathrm{p}}, \beta_{\mathrm{p}}, 0\right), \\
& b_{m^{\prime} n}^{\mathrm{T}}=\sum_{m=-n}^{n} B_{m n}^{\mathrm{T}} D_{m m^{\prime}}^{n}\left(\alpha_{\mathrm{p}}, \beta_{\mathrm{p}}, 0\right) .
\end{aligned}
$$


Similarly, in the global and particle coordinate systems, the scattered field is expanded in terms of radiating spherical vector wave functions:

$$
\begin{aligned}
\mathbf{E}_{\mathrm{sct}}(\mathbf{r}) & =\sum_{n=1}^{\infty} \sum_{m=-n}^{n} F_{m n} \mathbf{M}_{m n}^{3}\left(k_{\mathrm{o}} r, \theta, \varphi\right)+G_{m n} \mathbf{N}_{m n}^{3}\left(k_{\mathrm{o}} r, \theta, \varphi\right) \\
& =\sum_{n=1}^{\infty} \sum_{m=-n}^{n} f_{m n} \mathbf{M}_{m n}^{3}\left(k_{\mathrm{o}} r, \theta_{\mathrm{p}}, \varphi_{\mathrm{p}}\right)+g_{m n} \mathbf{N}_{m n}^{3}\left(k_{\mathrm{o}} r, \theta_{\mathrm{p}}, \varphi_{\mathrm{p}}\right),
\end{aligned}
$$

where

$$
\begin{aligned}
F_{m^{\prime} n} & =\sum_{m=-n}^{n} f_{m n} D_{m m^{\prime}}^{n}\left(0,-\beta_{\mathrm{p}}, \alpha_{\mathrm{p}}\right), \\
G_{m^{\prime} n} & =\sum_{m=-n}^{n} g_{m n} D_{m m^{\prime}}^{n}\left(0,-\beta_{\mathrm{p}}, \alpha_{\mathrm{p}}\right) .
\end{aligned}
$$

In addition to the fields described by Eqs. (11) and (16), a third field exists in the ambient medium. This field is called the interacting field and it is the result of the scattered field reflecting off the surface and striking the particle. In the global coordinate system, the interacting field can be expressed as

$$
\mathbf{E}_{\mathrm{sct}}^{\mathrm{R}}(\mathbf{r})=\sum_{n=1}^{\infty} \sum_{m=-n}^{n} F_{m n} \mathbf{M}_{m n}^{3 \mathrm{R}}\left(k_{\mathrm{o}} r, \theta, \varphi\right)+G_{m n} \mathbf{N}_{m n}^{3 \mathrm{R}}\left(k_{\mathrm{o}} r, \theta, \varphi\right)
$$

where $\mathbf{M}_{m n}^{3 \mathrm{R}}\left(k_{\mathrm{o}} r, \theta, \varphi\right)$ and $\mathbf{N}_{m n}^{3 \mathrm{R}}\left(k_{\mathrm{o}} r, \theta, \varphi\right)$ are the radiating spherical vector wave functions reflected by the plane surface. For $\mathbf{r}$ inside a sphere enclosed in the particle and a given azimuthal mode $m$, the reflected spherical vector wave functions can be expanded as

$$
\left(\begin{array}{c}
\mathbf{M}_{m n}^{3 \mathrm{R}}\left(k_{\mathrm{o}} r, \theta, \varphi\right) \\
\mathbf{N}_{m n}^{3 \mathrm{R}}\left(k_{\mathrm{o}} r, \theta, \varphi\right)
\end{array}\right)=\sum_{n^{\prime}=1}^{\infty}\left(\begin{array}{c}
\alpha_{m n n^{\prime}} \\
\gamma_{m n n^{\prime}}
\end{array}\right) \mathbf{M}_{m n^{\prime}}^{1}\left(k_{\mathrm{o}} r, \theta, \varphi\right)+\left(\begin{array}{c}
\beta_{m n n^{\prime}} \\
\delta_{m n n^{\prime}}
\end{array}\right) \mathbf{N}_{m n^{\prime}}^{1}\left(k_{\mathrm{o}} r, \theta, \varphi\right)
$$

whence inserting Eq. (20) into Eq. (19), we obtain the following series representation for the interacting field in terms of regular spherical vector wave functions:

$$
\mathbf{E}_{\mathrm{sct}}^{\mathrm{R}}(\mathbf{r})=\sum_{n^{\prime}=1}^{\infty} \sum_{m=-n^{\prime}}^{n^{\prime}} F_{m n^{\prime}}^{\mathrm{R}} \mathbf{M}_{m n^{\prime}}^{1}\left(k_{\mathrm{o}} r, \theta, \varphi\right)+G_{m n^{\prime}}^{\mathrm{R}} \mathbf{N}_{m n^{\prime}}^{1}\left(k_{\mathrm{o}} r, \theta, \varphi\right),
$$

where

$$
\left(\begin{array}{c}
F_{m n^{\prime}}^{\mathrm{R}} \\
G_{m n^{\prime}}^{\mathrm{R}}
\end{array}\right)=\sum_{n=1}^{\infty}\left(\begin{array}{c}
\alpha_{m n n^{\prime}} \\
\beta_{m n n^{\prime}}
\end{array}\right) F_{m n}+\left(\begin{array}{c}
\gamma_{m n n^{\prime}} \\
\delta_{m n n^{\prime}}
\end{array}\right) G_{m n}
$$

In Ref. [17], the expressions of the expansion coefficients $\alpha_{m n n^{\prime}}, \beta_{m n n^{\prime}}, \gamma_{m n n^{\prime}}$ and $\delta_{m n n^{\prime}}$ have been derived by making use on the integral representation of the radiating spherical vector vector wave functions; the results is

$$
\begin{aligned}
\alpha_{m n n^{\prime}} & =\frac{2 \mathrm{j}^{n^{\prime}-n}}{\sqrt{n n^{\prime}(n+1)\left(n^{\prime}+1\right)}} \int_{0}^{\pi / 2-\mathrm{j} \infty}\left[m^{2} \pi_{n}^{|m|}(\beta) \pi_{n^{\prime}}^{|m|}(\pi-\beta) r_{\|}(\beta)\right. \\
& \left.+\tau_{n}^{|m|}(\beta) \tau_{n^{\prime}}^{|m|}(\pi-\beta) r_{\perp}(\beta)\right] \mathrm{e}^{2 \mathrm{j} k_{\mathrm{o}} z_{0} \cos \beta} \sin \beta \mathrm{d} \beta, \\
\beta_{m n n^{\prime}} & =\frac{2 \mathrm{j}^{n^{\prime}-n}}{\sqrt{n n^{\prime}(n+1)\left(n^{\prime}+1\right)}} \int_{0}^{\pi / 2-\mathrm{j} \infty} m\left[\pi_{n}^{|m|}(\beta) \tau_{n^{\prime}}^{|m|}(\pi-\beta) r_{\|}(\beta)\right. \\
& \left.+\tau_{n}^{|m|}(\beta) \pi_{n^{\prime}}^{|m|}(\pi-\beta) r_{\perp}(\beta)\right] \mathrm{e}^{2 \mathrm{j} k_{0} z_{0} \cos \beta} \sin \beta \mathrm{d} \beta,
\end{aligned}
$$




$$
\begin{aligned}
\gamma_{m n n^{\prime}} & =\frac{2 \mathrm{j}^{n^{\prime}-n}}{\sqrt{n n^{\prime}(n+1)\left(n^{\prime}+1\right)}} \int_{0}^{\pi / 2-\mathrm{j} \infty} m\left[\tau_{n}^{|m|}(\beta) \pi_{n^{\prime}}^{|m|}(\pi-\beta) r_{\|}(\beta)\right. \\
& \left.+\pi_{n}^{|m|}(\beta) \tau_{n^{\prime}}^{|m|}(\pi-\beta) r_{\perp}(\beta)\right] \mathrm{e}^{2 \mathrm{j} k_{\mathrm{o}} z_{0} \cos \beta} \sin \beta \mathrm{d} \beta, \\
\delta_{m n n^{\prime}} & =\frac{2 \mathrm{j}^{n^{\prime}-n}}{\sqrt{n n^{\prime}(n+1)\left(n^{\prime}+1\right)}} \int_{0}^{\pi / 2-\mathrm{j} \infty}\left[\tau_{n}^{|m|}(\beta) \tau_{n^{\prime}}^{|m|}(\pi-\beta) r_{\|}(\beta)\right. \\
& \left.+m^{2} \pi_{n}^{|m|}(\beta) \pi_{n^{\prime}}^{|m|}(\pi-\beta) r_{\perp}(\beta)\right] \mathrm{e}^{2 \mathrm{j} k_{\mathrm{o}} z_{0} \cos \beta} \sin \beta \mathrm{d} \beta .
\end{aligned}
$$

Note that the integrals in Eqs. (23)-(26) are of the form

$$
I=\int_{0}^{\pi / 2-\mathrm{j} \infty} f(\cos \beta) \mathrm{e}^{2 \mathrm{j} q \cos \beta} \sin \beta \mathrm{d} \beta .
$$

By changing variables from $\beta$ to $x=-2 \mathrm{j} q(\cos \beta-1)$, we are led to the integrals

$$
I=\frac{e^{2 \mathrm{j} q}}{2 \mathrm{j} q} \int_{0}^{\infty} f\left(1-\frac{x}{2 \mathrm{j} q}\right) \mathrm{e}^{-x} \mathrm{~d} x
$$

which can be computed efficiently by using the Laguerre polynomials. In the particle coordinate system, the expansion of the interacting field reads as

$$
\mathbf{E}_{\mathrm{sct}}^{\mathrm{R}}(\mathbf{r})=\sum_{n^{\prime}=1}^{\infty} \sum_{m=-n^{\prime}}^{n^{\prime}} f_{m n^{\prime}}^{\mathrm{R}} \mathbf{M}_{m n^{\prime}}^{1}\left(k_{\mathrm{o}} r, \theta_{\mathrm{p}}, \varphi_{\mathrm{p}}\right)+g_{m n^{\prime}}^{\mathrm{R}} \mathbf{N}_{m n^{\prime}}^{1}\left(k_{\mathrm{o}} r, \theta_{\mathrm{p}}, \varphi_{\mathrm{p}}\right),
$$

with

$$
\begin{aligned}
f_{m^{\prime} n}^{\mathrm{R}} & =\sum_{m=-n}^{n} F_{m n}^{\mathrm{R}} D_{m m^{\prime}}^{n}\left(\alpha_{\mathrm{p}}, \beta_{\mathrm{p}}, 0\right), \\
g_{m^{\prime} n}^{\mathrm{R}} & =\sum_{m=-n}^{n} G_{m n}^{\mathrm{R}} D_{m m^{\prime}}^{n}\left(\alpha_{\mathrm{p}}, \beta_{\mathrm{p}}, 0\right) .
\end{aligned}
$$

Then, accounting of Eqs. (17), (18), (22), (28), and (29), we obtain the following representations of $f_{m n}^{\mathrm{R}}$ and $g_{m n}^{\mathrm{R}}$ in terms of $f_{m n}$ and $g_{m n}$ :

$$
\begin{aligned}
f_{m^{\prime} n^{\prime}}^{\mathrm{R}}=\sum_{n=1}^{\infty} \sum_{m=-n}^{n}\{ & {\left[\sum_{m^{\prime \prime}=-n^{\prime}}^{n^{\prime}} \alpha_{m^{\prime \prime} n n^{\prime}} D_{m m^{\prime \prime}}^{n}\left(0,-\beta_{\mathrm{p}}, \alpha_{\mathrm{p}}\right) D_{m^{\prime \prime} m^{\prime}}^{n^{\prime}}\left(\alpha_{\mathrm{p}}, \beta_{\mathrm{p}}, 0\right)\right] f_{m n} } \\
+ & {\left.\left[\sum_{m^{\prime \prime}=-n^{\prime}}^{n^{\prime}} \gamma_{m^{\prime \prime} n n^{\prime}} D_{m m^{\prime \prime}}^{n}\left(0,-\beta_{\mathrm{p}}, \alpha_{\mathrm{p}}\right) D_{m^{\prime \prime} m^{\prime}}^{n^{\prime}}\left(\alpha_{\mathrm{p}}, \beta_{\mathrm{p}}, 0\right)\right] g_{m n}\right\} } \\
g_{m^{\prime} n^{\prime}}^{\mathrm{R}}=\sum_{n=1}^{\infty} \sum_{m=-n}^{n}\{ & {\left[\sum_{m^{\prime \prime}=-n^{\prime}}^{n^{\prime}} \beta_{m^{\prime \prime} n n^{\prime}} D_{m m^{\prime \prime}}^{n}\left(0,-\beta_{\mathrm{p}}, \alpha_{\mathrm{p}}\right) D_{m^{\prime \prime} m^{\prime}}^{n^{\prime}}\left(\alpha_{\mathrm{p}}, \beta_{\mathrm{p}}, 0\right)\right] f_{m n} } \\
+ & {\left.\left[\sum_{m^{\prime \prime}=-n^{\prime}}^{n^{\prime}} \delta_{m^{\prime \prime} n n^{\prime}} D_{m m^{\prime \prime}}^{n}\left(0,-\beta_{\mathrm{p}}, \alpha_{\mathrm{p}}\right) D_{m^{\prime \prime} m^{\prime}}^{n^{\prime}}\left(\alpha_{\mathrm{p}}, \beta_{\mathrm{p}}, 0\right)\right] g_{m n}\right\} . }
\end{aligned}
$$

In the particle coordinate system, the scattered field coefficients are related to the expansion coefficients of the fields striking the particle through the transition matrix T. Truncating the expansions given by Eqs. (11), (16) and (21), we find the following matrix equation:

$$
\left[\begin{array}{l}
f_{m n} \\
g_{m n}
\end{array}\right]=\left[T_{m n, m^{\prime} n^{\prime}}\right]\left(\left[\begin{array}{c}
a_{m^{\prime} n^{\prime}}^{\mathrm{T}} \\
b_{m^{\prime} n^{\prime}}^{\mathrm{T}}
\end{array}\right]+\left[\begin{array}{c}
f_{m^{\prime} n^{\prime}}^{\mathrm{R}} \\
g_{m^{\prime} n^{\prime}}^{\mathrm{R}}
\end{array}\right]\right)
$$


Here, $n$ and $n^{\prime}$ range from 1 to $N_{\text {rank }}$, while $m$ and $m^{\prime}$ range from $-M_{\text {rank }}$ to $M_{\text {rank }}$, with $N_{\text {rank }}$ and $M_{\text {rank }}$ being the maximum expansion and azimuthal orders, respectively. In the particle coordinate system, the expansion coefficients of the interacting field are related to the scattered field coefficients by the so called reflection matrix:

$$
\left[\begin{array}{c}
f_{m^{\prime} n^{\prime}}^{\mathrm{R}} \\
g_{m^{\prime} n^{\prime}}^{\mathrm{R}}
\end{array}\right]=\left[R_{m n m^{\prime} n^{\prime}}\right]\left[\begin{array}{c}
f_{m n} \\
g_{m n}
\end{array}\right]
$$

where, in view of Eqs. (30) and (31), we have

$$
\left[R_{m n m^{\prime} n^{\prime}}\right]=\left[\begin{array}{ll}
\alpha_{m n m^{\prime} n^{\prime}} & \gamma_{m n m^{\prime} n^{\prime}} \\
\beta_{m n m^{\prime} n^{\prime}} & \delta_{m n m^{\prime} n^{\prime}}
\end{array}\right]
$$

with

$$
\alpha_{m n m^{\prime} n^{\prime}}=\sum_{m^{\prime \prime}=-n^{\prime}}^{n^{\prime}} \alpha_{m^{\prime \prime} n n^{\prime}} D_{m m^{\prime \prime}}^{n}\left(0,-\beta_{\mathrm{p}}, \alpha_{\mathrm{p}}\right) D_{m^{\prime \prime} m^{\prime}}^{n^{\prime}}\left(\alpha_{\mathrm{p}}, \beta_{\mathrm{p}}, 0\right)
$$

and similarly for $\beta_{m n m^{\prime} n^{\prime}}, \gamma_{m n m^{\prime} n^{\prime}}$ and $\delta_{m n m^{\prime} n^{\prime}}$. Now it is apparent that the scattered field coefficients $f_{m n}$ and $g_{m n}$ can be obtained by combining the matrix equations (32) and (33); we get

$$
\left(\mathrm{I}-\left[T_{m n, m^{\prime} n^{\prime}}\right]\left[R_{m n m^{\prime} n^{\prime}}\right]\right)\left[\begin{array}{c}
f_{m n} \\
g_{m n}
\end{array}\right]=\left[T_{m n, m^{\prime} n^{\prime}}\right]\left[\begin{array}{c}
a_{m^{\prime} n^{\prime}}^{T} \\
b_{m^{\prime} n^{\prime}}^{T}
\end{array}\right],
$$

where I is the identity matrix. It should be noted that for axisymmetric particles with arbitrary orientation, the scattering problem has to be solved simultaneously for all azimuthal modes. The matrices which enter in Eq. (36) are of dimenision $2 N_{\max } \times 2 N_{\max }$, where

$$
N_{\text {max }}=N_{\text {rank }}+M_{\text {rank }}\left(2 N_{\text {rank }}-M_{\text {rank }}+1\right) .
$$

For this reason, the computer code dealing with arbitrary particle orientation is more time consuming than the code dealing with particles having the axis of symmetry perpendicular to the plane surface. Once the scattered field coefficients in the particle coordinate system $f_{m n}$ and $g_{m n}$ have been computed, the scattered field coefficients in the global coordinate system $F_{m n}$ and $G_{m n}$ can be obtained from Eq. (17).

In the far-field region, the scattered field below the plane surface $(\pi / 2<\theta<\pi)$ sums the contribution of the direct electric far-field pattern $\mathbf{E}_{\mathrm{s} \infty}^{\mathrm{D}}(\theta, \varphi)$,

$$
\mathbf{E}_{\mathrm{s} \infty}^{\mathrm{D}}(\theta, \varphi)=\frac{1}{k_{\mathrm{o}}} \sum_{n=1}^{\infty} \sum_{m=-n}^{n}(-\mathrm{j})^{n+1}\left[F_{m n} \mathbf{m}_{m n}(\theta, \varphi)+\mathrm{j} G_{m n} \mathbf{n}_{m n}(\theta, \varphi)\right]
$$

and the interacting electric far-field pattern $\mathbf{E}_{\mathrm{s} \infty}^{\mathrm{R}}(\theta, \varphi)$,

$$
\mathbf{E}_{\mathrm{s} \infty}^{\mathrm{R}}(\theta, \varphi)=\frac{1}{k_{\mathrm{o}}} \sum_{n=1}^{\infty} \sum_{m=-n}^{n}(-\mathrm{j})^{n+1}\left[F_{m n} \mathbf{m}_{m n}^{\mathrm{R}}(\theta, \varphi)+\mathrm{j} G_{m n} \mathbf{n}_{m n}^{\mathrm{R}}(\theta, \varphi)\right],
$$

where $\mathbf{m}_{m n}$ and $\mathbf{n}_{m n}$ are the vector spherical harmonics, and $\mathbf{m}_{m n}^{\mathrm{R}}$ and $\mathbf{n}_{m n}^{\mathrm{R}}$ are the reflected vector spherical harmonics,

$$
\begin{aligned}
\mathbf{m}_{m n}^{\mathrm{R}}(\theta, \varphi) & =\frac{1}{\sqrt{2 n(n+1)}} \mathrm{e}^{-2 \mathrm{j} k_{\mathrm{o}} z_{0} \cos \theta}\left[\mathrm{j} m \pi_{n}^{|m|}(\theta) r_{\|}(\theta) \mathbf{e}_{\theta}-\tau_{n}^{|m|}(\theta) r_{\perp}(\theta) \mathbf{e}_{\varphi}\right] \mathrm{e}^{\mathrm{j} m \varphi}, \\
\mathbf{n}_{m n}^{\mathrm{R}}(\theta, \varphi) & =\frac{1}{\sqrt{2 n(n+1)}} \mathrm{e}^{-2 \mathrm{j} k_{\mathrm{o}} z_{0} \cos \theta}\left[\tau_{n}^{|m|}(\theta) r_{\|}(\theta) \mathbf{e}_{\theta}+\mathrm{j} m \pi_{n}^{|m|}(\theta) r_{\perp}(\theta) \mathbf{e}_{\varphi}\right] \mathrm{e}^{\mathrm{j} m \varphi} .
\end{aligned}
$$

In Eqs. (39) and (40), $r_{\|}$and $r_{\perp}$ are the Fresnel reflection coefficients given by

$$
\begin{aligned}
& r_{\|}(\theta)=\frac{m_{\mathrm{rs}} \cos \theta-\cos \theta_{\mathrm{s}}}{m_{\mathrm{rs}} \cos \theta+\cos \theta_{\mathrm{s}}}, \\
& r_{\perp}(\theta)=\frac{\cos \theta-m_{\mathrm{rs}} \cos \theta_{\mathrm{s}}}{\cos \theta+m_{\mathrm{rs}} \cos \theta_{\mathrm{s}}},
\end{aligned}
$$

with $\sin \theta_{\mathrm{s}}=\left(1 / m_{\mathrm{rs}}\right) \sin \theta$.

Thus, the solution of the scattering problem in the framework of the separation of variables method involves the following steps: 
1. calculation of the $T$ matrix relating the expansion coefficients of the fields striking the particle to the scattered field coefficients;

2. calculation of the reflection matrix $\mathrm{R}$ characterizing the reflection of spherical vector wave functions by the surface;

3. computation of an approximate solution by solving the matrix equation (36);

4. computation of the far-field pattern by using (37) and (38).

\section{$2.2 \quad$ Imaging of the scattered light}

Calculation of the image of the scattered field involves the following steps:

1. computation of the scattered field on the Gaussian reference sphere of the collector lens,

2. computation of the transmitted field on the Gaussian reference sphere of the detector lens, and

3. computation of the focus field by means of the Debye diffraction integral.

Before proceeding we note that the aperture angles in the object and image spaces, i.e., the polar angles under which the Gaussian reference spheres are observed at the focii of the collector and detector lens, respectively, are computed as

$$
\begin{aligned}
& \Theta_{\mathrm{o}}=\arcsin \left(\mathrm{NA}_{\mathrm{o}} / n_{\mathrm{o}}\right), \\
& \Theta_{\mathrm{i}}=\arctan \left(\frac{f_{\mathrm{o}}}{f_{\mathrm{i}}} \tan \Theta_{\mathrm{o}}\right),
\end{aligned}
$$

where $\mathrm{NA}_{\mathrm{o}}$ is the numerical aperture of the collector lens and $\mathrm{NA}_{\mathrm{i}}=n_{\mathrm{i}} \sin \Theta_{\mathrm{i}}$ is the numerical aperture of the detector lens.

Step 1. The scattered field on the Gaussian reference sphere of the collector lens is given by

$$
\mathbf{E}_{\mathrm{s}}(\theta, \varphi)=E_{\mathrm{s} \theta}(\theta, \varphi) \mathbf{e}_{\theta}+E_{\mathrm{s} \varphi}(\theta, \varphi) \mathbf{e}_{\varphi},
$$

where

$$
\begin{aligned}
& E_{\mathrm{s} \theta}(\theta, \varphi)=\frac{\mathrm{e}^{\mathrm{j} k_{\mathrm{o}} f_{\mathrm{o}}}}{f_{\mathrm{o}}}\left[E_{\mathrm{s} \infty \theta}^{\mathrm{D}}(\theta, \varphi)+E_{\mathrm{s} \infty \theta}^{\mathrm{R}}(\theta, \varphi)\right], \\
& E_{\mathrm{s} \varphi}(\theta, \varphi)=\frac{\mathrm{e}^{\mathrm{j} k_{\mathrm{o}} f_{\mathrm{o}}}}{f_{\mathrm{o}}}\left[E_{\mathrm{s} \infty \varphi}^{\mathrm{D}}(\theta, \varphi)+E_{\mathrm{s} \infty \varphi}^{\mathrm{R}}(\theta, \varphi)\right],
\end{aligned}
$$

while the so-called integral response of the detector is computed as

$$
P=f_{\mathrm{o}}^{2} \int_{0}^{2 \pi} \int_{0}^{\Theta_{\mathrm{o}}}\left[\left|E_{\mathrm{s} \theta}(\theta, \varphi)\right|^{2}+\left|E_{\mathrm{s} \varphi}(\theta, \varphi)\right|^{2}\right] \sin \theta \mathrm{d} \theta \mathrm{d} \varphi .
$$

Step 2. The transmitted field in the image space can be computed by (vector) ray tracing. We assume that a ray propagating in the object space in the direction $(\theta, \varphi)$ is deflected into a ray that propagates in the image space in the direction $\left(\theta_{\mathrm{i}}, \varphi_{\mathrm{i}}\right)$, where

$$
\begin{aligned}
\theta_{\mathrm{i}} & =\arctan \left[\left(f_{\mathrm{o}} / f_{\mathrm{i}}\right) \tan (\theta-\pi)\right], \\
\varphi_{\mathrm{i}} & =\psi_{\mathrm{i}}+\pi=3 \pi / 2-\varphi .
\end{aligned}
$$

Consequently, the polar unit vectors $\mathbf{e}_{\theta}$ and $\mathbf{e}_{\varphi}$ are deflected into the polar unit vectors $\mathbf{e}_{\theta \mathrm{i}}$ and $\mathbf{e}_{\varphi \mathrm{i}}$ given by

$$
\begin{aligned}
\mathbf{e}_{\theta \mathrm{i}} & =\cos \theta_{\mathrm{i}} \cos \varphi_{\mathrm{i}} \mathbf{e}_{\mathrm{xi}}+\cos \theta_{\mathrm{i}} \sin \varphi_{\mathrm{i}} \mathbf{e}_{\mathrm{yi}}-\sin \theta_{\mathrm{i}} \mathbf{e}_{\mathrm{zi}} \\
\mathbf{e}_{\varphi \mathrm{i}} & =-\sin \varphi_{\mathrm{i}} \mathbf{e}_{\mathrm{xi}}+\cos \varphi_{\mathrm{i}} \mathbf{e}_{\mathrm{yi}},
\end{aligned}
$$


where $\left(\mathbf{e}_{\mathrm{xi}}, \mathbf{e}_{\mathrm{yi}}, \mathbf{e}_{\mathrm{zi}}\right)$ are the Cartesian unit vectors in the global coordinate system $O_{\mathrm{i}} x_{\mathrm{i}} y_{\mathrm{i}} z_{\mathrm{i}}$ centered at the focal point of the detector lens. The transmitted field on the Gaussian reference sphere of the detector lens is then written as

$$
\mathbf{E}_{\mathrm{si}}\left(\theta_{\mathrm{i}}, \varphi_{\mathrm{i}}\right)=T_{\theta} E_{\mathrm{s} \theta}\left(\theta_{\mathrm{i}}, \varphi_{\mathrm{i}}\right) \mathbf{e}_{\theta \mathrm{i}}+T_{\varphi} E_{\mathrm{s} \varphi}\left(\theta_{\mathrm{i}}, \varphi_{\mathrm{i}}\right) \mathbf{e}_{\varphi \mathrm{i}}
$$

where $T_{\theta}=T_{\theta}\left(\theta_{\mathrm{i}}, \varphi_{\mathrm{i}}\right)$ and $T_{\varphi}=T_{\varphi}\left(\theta_{\mathrm{i}}, \varphi_{\mathrm{i}}\right)$ are the transmission coefficients (pupil function, apodization) for parallel and perpendicular polarization, respectively. Accumulated phase distortions, i.e. aberrations at the principal plane of the detector lens, as well as attenuations, i.e. amplitude factors, are integrated in the complex parameters $T_{\theta}$ and $T_{\varphi}$. The computation of $T_{\theta}$ and $T_{\varphi}$ requires the knowledge of the imaging system and is based on the Fresnel equations. In our model, $T_{\theta}$ and $T_{\varphi}$ are input parameters.

Step 3. In microscopy, the plane wave spectrum method is the essence of the Debye approximation and is often used for the calculation of the electromagnetic field near the focus of high numerical aperture objectives. However, for optical systems with high numerical aperture, this classical problem turns into a computational challenge due to the highly oscillatory behavior of the involved functions. A novel and flexible implementation of the Debye integral incorporating the effects of amplitude, phase and polarization in an overall manner has been proposed in Ref. [18]. Instead of direct integration, the vector Debye diffraction integral is evaluated in the entire focal region with the fast Fourier transform. This implementation which is used in our derivation, is summarized below.

In the Debye approximation, the transmitted field $\mathbf{E}_{\mathrm{si}}$ as given by Eq. (49) is the plane wave spectrum of the field $\mathbf{E}$ near the focus of the detector lens. Hence, the electric field $\mathbf{E}$ at a point $\left(x_{\mathrm{i}}, y_{\mathrm{i}}, z_{\mathrm{i}}\right)$ is obtained by integrating the propagating plane waves, i.e.,

$$
\mathbf{E}\left(x_{\mathrm{i}}, y_{\mathrm{i}}, z_{\mathrm{i}}\right)=-\mathrm{j} \frac{f_{\mathrm{i}}}{\lambda_{0}} \int_{0}^{2 \pi} \int_{0}^{\Theta_{\mathrm{i}}} \mathbf{E}_{\mathrm{si}}\left(\theta_{\mathrm{i}}, \varphi_{\mathrm{i}}\right) \mathrm{e}^{\mathrm{j}\left(k_{\mathrm{ix}} x_{\mathrm{i}}+k_{\mathrm{i}} y_{\mathrm{i}}+k_{\mathrm{iz}} z_{\mathrm{i}}\right)} \sin \theta_{\mathrm{i}} \mathrm{d} \theta_{\mathrm{i}} \mathrm{d} \varphi_{\mathrm{i}}
$$

where $\Theta_{\mathrm{i}}$ is the aperture angle of the detector lens, and

$$
\begin{aligned}
& k_{\mathrm{ix}}=k_{\mathrm{i}} \sin \theta_{\mathrm{i}} \cos \varphi_{\mathrm{i}}, \\
& k_{\mathrm{iy}}=k_{\mathrm{i}} \sin \theta_{\mathrm{i}} \sin \varphi_{\mathrm{i}}, \\
& k_{\mathrm{iz}}=k_{\mathrm{i}} \cos \theta_{\mathrm{i}},
\end{aligned}
$$

with $k_{\mathrm{i}}=k_{0} n_{\mathrm{i}}$. Using the representations (51) and (52), we express the integral in Eq. (50) as

$$
\mathbf{E}\left(x_{\mathrm{i}}, y_{\mathrm{i}}, z_{\mathrm{i}}\right)=-\mathrm{j} \frac{f_{\mathrm{i}}}{\lambda_{0} k_{\mathrm{i}}^{2}} \int_{D_{\mathrm{k}}} \frac{\mathbf{E}_{\mathrm{si}}\left(\theta_{\mathrm{i}}, \varphi_{\mathrm{i}}\right)}{\cos \theta_{\mathrm{i}}} \mathrm{e}^{\mathrm{j} k_{\mathrm{i}} z_{\mathrm{i}} \cos \theta_{\mathrm{i}}} \mathrm{e}^{\mathrm{j}\left(k_{\mathrm{ix}} x_{\mathrm{i}}+k_{\mathrm{iy}} y_{\mathrm{i}}\right)} \mathrm{d} k_{\mathrm{ix}} \mathrm{d} k_{\mathrm{iy}},
$$

with

$$
D_{\mathrm{k}}=\left\{\left(k_{\mathrm{ix}}, k_{\mathrm{iy}}\right) \mid \sqrt{k_{\mathrm{ix}}^{2}+k_{\mathrm{iy}}^{2}} \leq K\right\}
$$

and $K=k_{\mathrm{i}} \sin \Theta_{\mathrm{i}}=k_{0} \mathrm{NA}_{\mathrm{i}}$. For an equidistant sampling $k_{\mathrm{ix}}=m \triangle K$ and $k_{\mathrm{iy}}=n \triangle K$, where $\triangle K=K / M, M$ is the number of sampling points over $K$, and $m, n=-M, \ldots, M$, the sampling polar angles are

$$
\begin{aligned}
& \theta_{\mathrm{i} m n}=\arcsin \left(\frac{\triangle K}{k_{\mathrm{i}}} \sqrt{m^{2}+n^{2}}\right), \\
& \varphi_{\mathrm{i} m n}=\arccos \left(\frac{m}{\sqrt{m^{2}+n^{2}}}\right),
\end{aligned}
$$

and the numerical implementation of the integral (54) is

$$
\begin{aligned}
\mathbf{E}\left(x_{\mathrm{i}}, y_{\mathrm{i}}, z_{\mathrm{i}}\right) & =-\mathrm{j} \frac{f_{\mathrm{i}} \triangle K^{2}}{\lambda_{0} k_{\mathrm{i}}^{2}} \sum_{m=-M}^{M} \sum_{n=-M}^{M} \frac{\mathbf{E}_{\mathrm{ii}}\left(\theta_{\mathrm{i} m n}, \varphi_{\mathrm{i} m n}\right)}{\cos \theta_{\mathrm{i} m n}} \\
& \times \mathrm{e}^{\mathrm{j} k_{\mathrm{i}} z_{\mathrm{i}} \cos \theta_{\mathrm{i} m n}} \mathrm{e}^{\mathrm{j} \triangle K\left(m x_{\mathrm{i}}+n y_{\mathrm{i}}\right)}
\end{aligned}
$$

with

$$
\mathbf{E}_{\mathrm{si}}\left(\theta_{\mathrm{i} m n}, \varphi_{\mathrm{i} m n}\right)=0 \text { for } \sqrt{m^{2}+n^{2}}>M .
$$

A fast Fourier transform (FFT) is then used for computation of the double sum in Eq. (58). For this purpose, we choose the number of FFT sampling points per transformed dimension $N=2^{s}$, where $s$ is an integer, such 
that the condition $N \geq 4 M$ is satisfied. This condition can be explained as follows. Due to the Debye diffraction integral, the field $\mathbf{E}_{\mathrm{si}}$ is the plane wave spectrum of the field $\mathbf{E}$, and usually, the smallest area (aperture matrix) containing $\mathbf{E}_{\mathrm{si}} \neq 0$ is transformed. The spectral product $\exp \left(\mathrm{j} k_{\mathrm{iz}} z_{\mathrm{i}}\right) \mathbf{E}_{\mathrm{si}} / \cos \theta_{\mathrm{i}}$ represents the spatial convolution $\mathcal{F}\left(\exp \left(\mathrm{j} k_{\mathrm{iz}} z_{\mathrm{i}}\right)\right) \star \mathcal{F}\left(\mathbf{E}_{\mathrm{si}} / \cos \theta_{\mathrm{i}}\right)$, where $\mathcal{F}$ denotes the Fourier transform. In general, the result of the convolution is non-zero on an area larger than the aperture size, which may cause aliasing. Therefore, the aperture matrix is enlarged by zero padding to at least twice its dimensions $(N \geq 2(2 M))$ before performing the transform. In a final step, simple cropping of the transform output removes the padding. Mathematically this process can be described as follows:

1. for $m_{1}, n_{1}=0, \ldots, N-1$, compute the aperture matrix as

$$
\begin{aligned}
\mathbf{E}_{\mathrm{A} m_{1} n_{1}}\left(z_{\mathrm{i}}\right) & =-\mathrm{j} \frac{f_{\mathrm{i}} \triangle K^{2}}{\lambda_{0} k_{\mathrm{i}}^{2}} \frac{\mathbf{E}_{\mathrm{si}}\left(\theta_{\mathrm{i} m_{1}-M n_{1}-M}, \varphi_{\mathrm{i} m_{1}-M n_{1}-M}\right)}{\cos \theta_{\mathrm{i} m_{1}-M n_{1}-M}} \\
& \times \mathrm{e}^{\mathrm{j} k_{\mathrm{i}} z_{\mathrm{i}} \cos \theta_{\mathrm{i} m_{1}-M n_{1}-M}},
\end{aligned}
$$

with

$$
\mathbf{E}_{\mathrm{A} m_{1} n_{1}}\left(z_{\mathrm{i}}\right)=0 \text { for } \sqrt{\left(m_{1}-M\right)^{2}+\left(n_{1}-M\right)^{2}}>M
$$

2. compute the Fourier-transformed matrix

$$
\mathbf{E}_{\mathrm{F} m_{1} n_{1}}\left(z_{\mathrm{i}}\right)=\mathcal{F}\left(\mathbf{E}_{\mathrm{A} m_{1} n_{1}}\left(z_{\mathrm{i}}\right)\right) ;
$$

3. for $m_{2}, n_{2}=-N / 2+1, \ldots, N / 2$, compute the focus field $\mathbf{E}$ at the sampling points $x_{\mathrm{i} m_{2}}=m_{2} \Delta x$ and $y_{\mathrm{in} n_{2}}=n_{2} \triangle y$ as

$$
\mathbf{E}\left(x_{\mathrm{i} m_{2}}, y_{\mathrm{i} m_{2}}, z_{\mathrm{i}}\right)=\mathbf{E}_{\mathrm{F} m_{2}+N / 2-1 n_{2}+N / 2-1}\left(z_{\mathrm{i}}\right),
$$

where

$$
\triangle x=\triangle y=\frac{2 \pi}{N \triangle K}
$$

4. crop the matrix $\mathbf{E}\left(x_{\mathrm{i} m_{2}}, y_{\mathrm{i} m_{2}}, z_{\mathrm{i}}\right)$ by restricting $m_{2}$ and $n_{2}$ to vary in the range $m_{2}, n_{2}=-N_{\mathrm{c}} / 2+1, \ldots, N_{\mathrm{c}} / 2$, where $N_{\mathrm{c}}<N$.

Along the $z_{\mathrm{i}}$-direction, the sampling can be chosen arbitrarily by respecting the condition

$$
M>\frac{2\left(\mathrm{NA}_{\mathrm{i}}\right)^{2}}{\sqrt{n_{\mathrm{i}}^{2}-\left(\mathrm{NA}_{\mathrm{i}}\right)^{2}}} \frac{\left|z_{\mathrm{i}}\right|}{\lambda_{0}} .
$$

Condition (60) also known as the sampling condition guarantees that the propagation factor $\exp \left(\mathrm{j} k_{\mathrm{i}} z_{\mathrm{i}} \cos \theta_{\mathrm{i}}\right)$ is calculated with high resolution. In particular, the phase term $k_{\mathrm{i}} z_{\mathrm{i}} \cos \theta_{\mathrm{i}}$ does not change by more than $\pi$ between neighboring sampling points in the Gaussian reference sphere. In addition, a lower limit of $M>50$ was found to be necessary for an accurate sampling of $\varphi_{\mathrm{i}}$. Deviations from these sampling conditions result in granular artifacts in the final computed image. As a typical value for $M$, we chose $M=120$, while for the axial distance $z_{\mathrm{i}}$, we impose $\left|z_{\mathrm{i}}\right|<25 \lambda_{0}$, corresponding to $\left|z_{\mathrm{i}}\right|<16 \mu \mathrm{m}$ at a wavelength of $635 \mathrm{~nm}$. The final result is the distribution of the intensity

$$
I_{m_{2} n_{2}}\left(z_{\mathrm{i}}\right)=\left|E_{\mathrm{x}}\left(x_{\mathrm{i} m_{2}}, y_{\mathrm{i} m_{2}}, z_{\mathrm{i}}\right)\right|^{2}+\left|E_{\mathrm{y}}\left(x_{\mathrm{i} m_{2}}, y_{\mathrm{i} m_{2}}, z_{\mathrm{i}}\right)\right|^{2}+\left|E_{\mathrm{z}}\left(x_{\mathrm{i} m_{2}}, y_{\mathrm{i} m_{2}}, z_{\mathrm{i}}\right)\right|^{2},
$$

at the sampling points $\left(x_{\mathrm{i} m_{2}}=m_{2} \triangle x, y_{\mathrm{i} n_{2}}=n_{2} \triangle y\right)$ in the axial plane $z_{\mathrm{i}}$.

\section{Numerical Simulations}

In our numerical simulations we choose the wavelength in free space $\lambda_{0}=0.635 \mu \mathrm{m}$, the refractive index in the object space (water) $n_{\mathrm{o}}=1.33$, the refractive index of the particle $n_{\mathrm{p}}=1.591$, the refractive index of the substrate (glass) $n_{\mathrm{s}}=1.51$, the refractive index in the image space (water) $n_{\mathrm{i}}=1.33$, the transversal magnification $m_{\mathrm{T}}=f_{\mathrm{i}} / f_{\mathrm{o}}=60$, and the numerical aperture in the object space $\mathrm{NA}_{\mathrm{o}}=1.0$. The polarization angle of the incident wave is $\alpha_{\text {pol }}=45^{\circ}$.

First we analyze the accuracy of the newly developed code devoted to the scattering analysis of axiysmmetric particles with arbitrary orientation. For this purpose we take the results obtained for a spherical particle as in Ref. [17]. In the new code, the spherical particle is treated as an axisymmetric particle, and the parameters of 


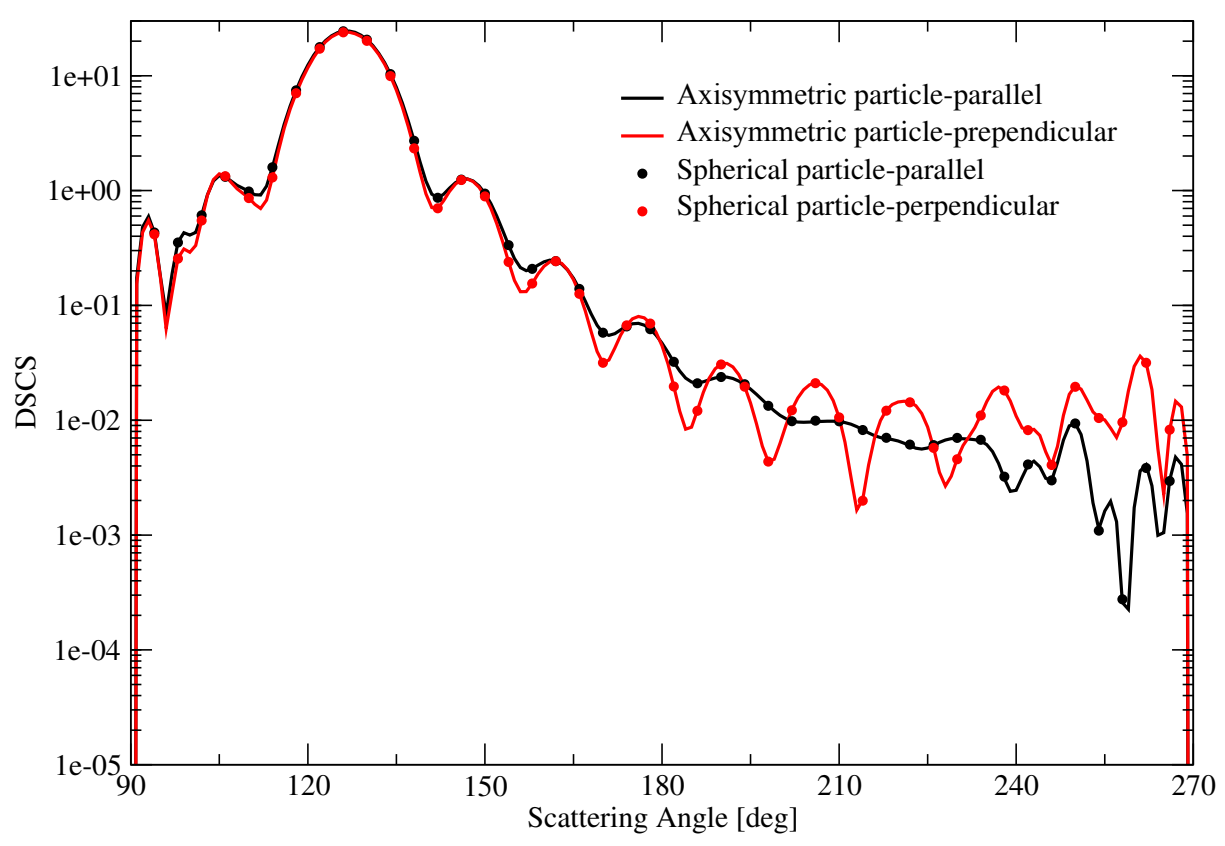

Figure 2: Differential scattering cross sections for parallel and perpendicular polarization of a homogeneous spherical particle with $a=b=1 \mu \mathrm{m}$.

calculation are chosen as follows: $a=b=1.0 \mu \mathrm{m}, \alpha_{\mathrm{p}}=30^{\circ}, \beta_{\mathrm{p}}=45^{\circ}$, and $\beta_{\mathrm{s}}=45^{\circ}$. By this choice of the particle orientation angles (i.e., $\alpha_{\mathrm{p}}=30^{\circ}$ and $\beta_{\mathrm{p}}=45^{\circ}$ ) we check the correctness of the new implementation based on the rotation addition theorem. The results illustrated in Fig. 2 show a complete agreement between the scattering curves.

In Fig. 3 we show the focus intensity distribution along the $x_{\mathrm{i}}$-axis for an axisymmetric particle with $a=1.0 \mu \mathrm{m}$ and $b=0.5 \mu \mathrm{m}$. The original image $I_{m_{2} n_{2}}(0)$ with $N=512$ is first cropped by considering the image $I_{m_{2} n_{2}}(0)$ with $N_{\mathrm{c}}=128$, and then filtered out by setting $I_{m_{2} n_{2}}(0)=0$ if $I_{m_{2} n_{2}}(0)<\varepsilon I_{\max }(0)$, where $I_{\max }(0)=\max _{m_{2}, n_{2}} I_{m_{2} n_{2}}(0)$ and $\varepsilon=10^{-3}$. In this simulation and the subsequent ones, we choose $M=120$, so that the condition $N>4 M$ is satisfied.

From our theoretical analysis we know that if $\beta_{\mathrm{s}}<\beta_{\mathrm{sc}}$, the particle is illuminated by a propagating wave, while in the converse situation, namely when $\beta_{\mathrm{s}}>\beta_{\mathrm{sc}}$, the particle is illuminated by an evanescent wave. Note that for the scattering problem under examination, the critical angle of total internal reflection (after which evanescent waves appear) is

$$
\beta_{\mathrm{sc}}=\arcsin \left(\frac{n_{\mathrm{o}}}{n_{\mathrm{s}}}\right) \approx 61.73^{\circ} .
$$

In Fig. 4 we illustrate the focus intensity distributions for a particle placed at an effective distance $d=0.1 \mu \mathrm{m}$ with respect to the plane surface, and being illuminated at two angles of incidence: $\beta_{\mathrm{s}}=0^{\circ}<\beta_{\mathrm{sc}}$ and $\beta_{\mathrm{s}}=62^{\circ}>\beta_{\mathrm{sc}}$. In the first case, the image of the particle has a good contrast, while in the second case, the image is blurry. In Table 1 we show the integral response of the same particle for three values of the incident angle $\beta_{\mathrm{s}}$, and two values of the effective distance between the particle and the plane surface $d$. As it can be seen, for $\beta_{\mathrm{s}}<\beta_{\mathrm{sc}}$, the integral response is insensitive with respect to $d$, while for $\beta_{\mathrm{s}}>\beta_{\mathrm{sc}}$, the integral response decreases by a factor of 5 when $d$ increases from $0.1 \mu \mathrm{m}$ to $1.0 \mu \mathrm{m}$.

The plots in Figs. 5 and 6 confirm the above findings. For $\beta_{\mathrm{s}}<\beta_{\mathrm{sc}}$, the image in Fig. 5, with an excellent quality in terms of sharpness and contrast, carries information on the size and the orientation of the particle, while for $\beta_{\mathrm{s}}>\beta_{\mathrm{sc}}$, the image in Fig. 6 is blurry. However, in the second case, the integral response $P$ plotted in Fig. 7 contains information about the effective distance $d$. In fact, these combination of figures demonstrate an approach for determining the impact of collecting scattered light from an image [11, 13], as is done with a digital camera. Experiments consist of collecting images similar to those shown in Fig. 5 with subsequent integration, as is shown in Fig. 7. Note, however, that the influence of the camera itself has not been included.

Referring again to Fig. 7, a rough estimate can be made: if $P$ can be measured with a resolution of $1 / \triangle P$, where $\triangle P=P_{\max }-P_{\min } \approx 2.8$, then $d$ can be measured with a resolution of about $10 \mathrm{~nm}$. Moreover, it is apparent that the integral response decays exponentially with the distance. Fitting the model data with the exponential curve 

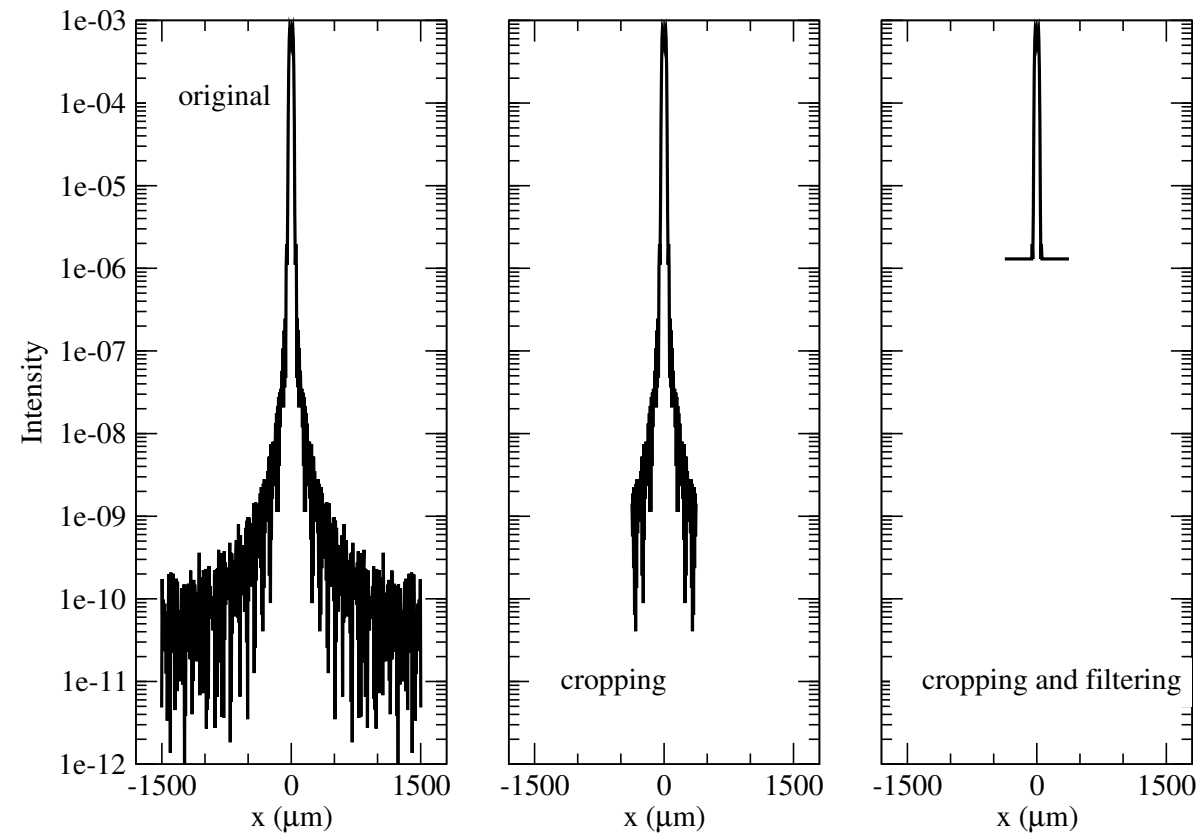

Figure 3: The focus intensity distribution along the $x_{\mathrm{i}}$-axis. The original image is first cropped and then filtered.

Table 1: Integral response of a particle with $a=1.5 \mu \mathrm{m}, b=0.5 \mu \mathrm{m}, \alpha_{\mathrm{p}}=45^{\circ}$, and $\beta_{\mathrm{p}}=90^{\circ}$ for three values of the incident angle $\beta_{\mathrm{s}}$, and two values of the effective distance between the particle and the plane surface $d$.

\begin{tabular}{lll}
\hline$\beta_{\mathrm{s}}$ & $d=0.1 \mu \mathrm{m}$ & $d=1.0 \mu \mathrm{m}$ \\
\hline $0^{\circ}$ & $1.07 \mathrm{E}+03$ & $1.07 \mathrm{E}+03$ \\
$45^{\circ}$ & $3.53 \mathrm{E}+02$ & $3.52 \mathrm{E}+02$ \\
$62^{\circ}$ & $4.08 \mathrm{E}+00$ & $7.5 \mathrm{E}-01$ \\
\hline
\end{tabular}

$P(d=0) \exp (-d / \rho)$, we found that the decay length is $\rho=0.55 \mu \mathrm{m}$. This value is very close to the penetration depth of the evanescent wave $\rho_{0}$, estimated as [19]

$$
\rho_{0}=\frac{\lambda_{0}}{4 \pi \sqrt{\left(n_{\mathrm{s}} \sin \beta_{\mathrm{s}}\right)^{2}-n_{\mathrm{o}}^{2}}} \approx 0.543 \mu \mathrm{m} .
$$

Experimental application of the model to TIRM will require an apparatus utilizing a digital camera, rather than a photomultiplier tube. The camera will capture images of the morphology of evanescent wave scattering from a particle. As shown above, the scattering morphology contains information related to the separation distance, orientation, and shape of the particle. Morphology mapping simulations will be paired with experiments, in which images of scattering from particles of known orientation, separation distance, and shape will be obtained. These maps will provide not only experimental verification of the model, but also a reference, either via direct comparison or analytical expression, for the measurement of a particle of unknown orientation and separation distance. Previous work has shown how to subsequently assemble a potential energy landscape from these separation distance and orientation observations [20]. We suggest the name for the technique, where scattering morphology (instead of the integrated intensity) is used to calculate particle position and orientation, as 'Scattering Morphology Resolved Total Internal Reflection Microscopy (SMR - TIRM)'.

\section{Conclusions}

In this paper a light scattering model for TIRM is described. The model deals with scattering by an axisymmetric particle of arbitrary orientation situated near a plane surface, and imaging of the scattered light. Scattering by an axisymmetric particle of arbitrary orientation situated near a plane surface is analyzed by using the T-matrix method and the rotation addition theorem for spherical vector wave functions. Essentially, this theorem enabled us to pass from the field expansions in the global coordinate system to the field expansions in the particle coordinate 


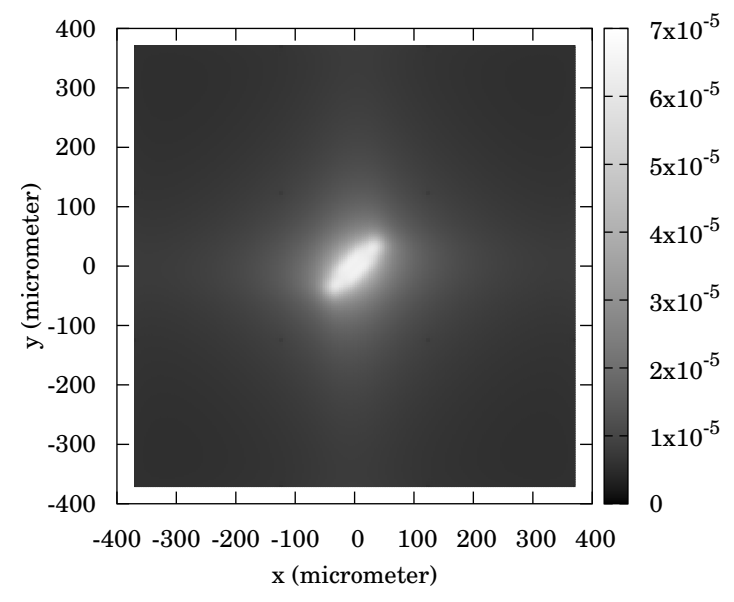

(a)

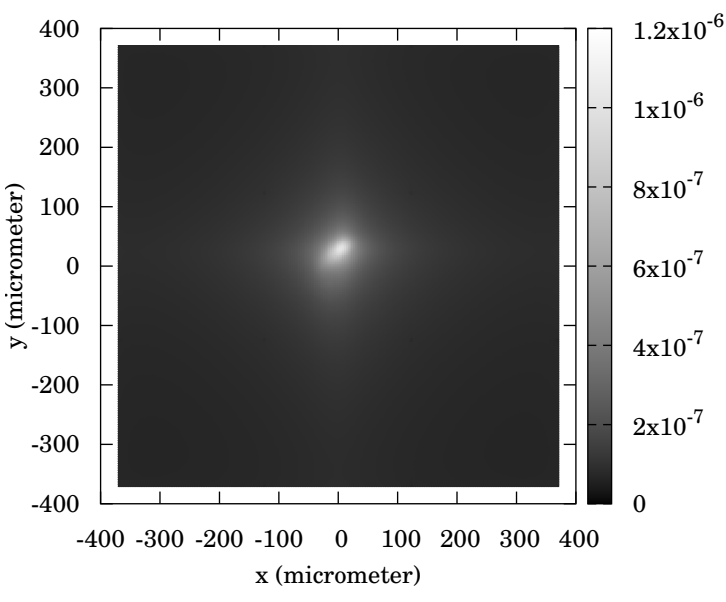

(b)

Figure 4: The focus intensity distributions for a particle with $a=1.5 \mu \mathrm{m}, b=0.5 \mu \mathrm{m}, \alpha_{\mathrm{p}}=45^{\circ}$, and $\beta_{\mathrm{p}}=90^{\circ}$. The incident angle is $\beta_{\mathrm{s}}=0^{\circ}$ (a) and $\beta_{\mathrm{s}}=62^{\circ}$ (b). The effective distance between the particle and the plane surface is $d=0.1 \mu \mathrm{m}$.

system and vice versa. A direct consequence of the arbitrary particle orientation is that the scattering problem does not decouple over the azimuthal modes as it happens when the axis of symmetry of the particle is perpendicular to the plane surface. The computation of the image of the scattered field has been performed in three steps: (i) computation of the scattered field on the Gaussian reference sphere of the collector lens, (ii) computation of the transmitted field on the Gaussian reference sphere of the detector lens, and (iii) computation of the focus field by means of the Debye diffraction integral.

Our numerical simulations indicated that two working regimes for TIRM can be identified.

1. The first regime, corresponding to an incident angle less than the critical angle of total internal reflection, gives information on the size and the orientation of the particle; the computed images have a good contrast and the particle shape is clearly visualized.

2. The second regime, corresponding to an incident angle larger than the critical angle of total internal reflection, is recommended for measuring the effective distance between the particle and plane surface. As a matter of fact, it seems that TIRM offers the possibility to measure effective distances with a resolution of $10 \mathrm{~nm}$.

\section{Acknowledgement}

Christopher L. Wirth was supported by the Cleveland State University Office of Research Startup Fund and the National Science Foundation CAREER Award no. 1752051.

\section{References}

[1] Dennis C. Prieve, Foo Luo, and Frederick Lanni. Brownian motion of a hydrosol particle in a colloidal force field. Faraday Discussions of the Chemical Society, 83:297, 1987.

[2] H. H. von Grünberg, L. Helden, P. Leiderer, and C. Bechinger. Measurement of surface charge densities on brownian particles using total internal reflection microscopy. The Journal of Chemical Physics, 114(22):1009410104, jun 2001.

[3] D. C. Prieve, J. D. Hoggard, R. Fu, P. J. Sides, and R. Bethea. Two independent measurements of debye lengths in doped nonpolar liquidst. Langmuir, 24(4):1120-1132, feb 2008. 


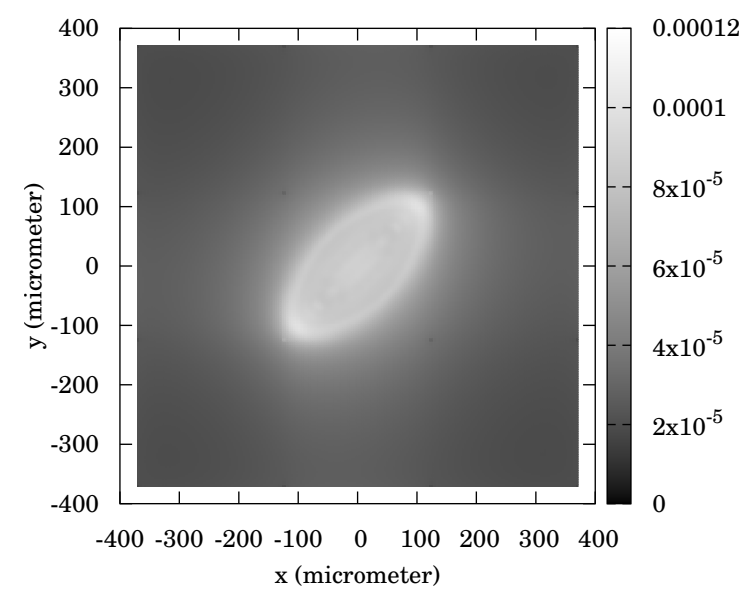

(a)

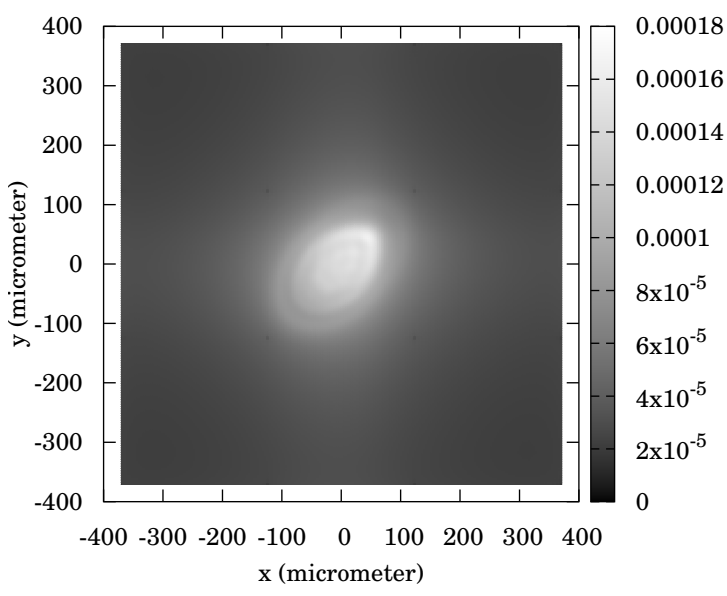

(b)

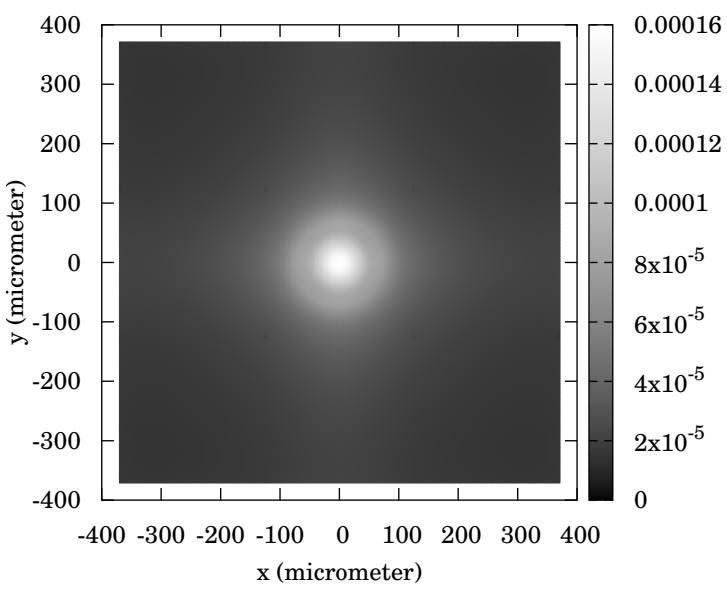

(c)

Figure 5: The focus intensity distribution for a particle with $a=3.0 \mu \mathrm{m}$ and $b=1.5 \mu \mathrm{m}$. The incident angle is $\beta_{\mathrm{s}}=0^{\circ}$, and the effective distance between the particle and the plane surface is $d=0.1 \mu \mathrm{m}$. The azimuth orientation angle of the particle is $\alpha_{\mathrm{p}}=45^{\circ}$, while the zenith orientation angle are $\beta_{\mathrm{p}}=90^{\circ}(\mathrm{a}), \beta_{\mathrm{p}}=45^{\circ}(\mathrm{b})$, and $\beta_{\mathrm{p}}=0^{\circ}$ (c). 


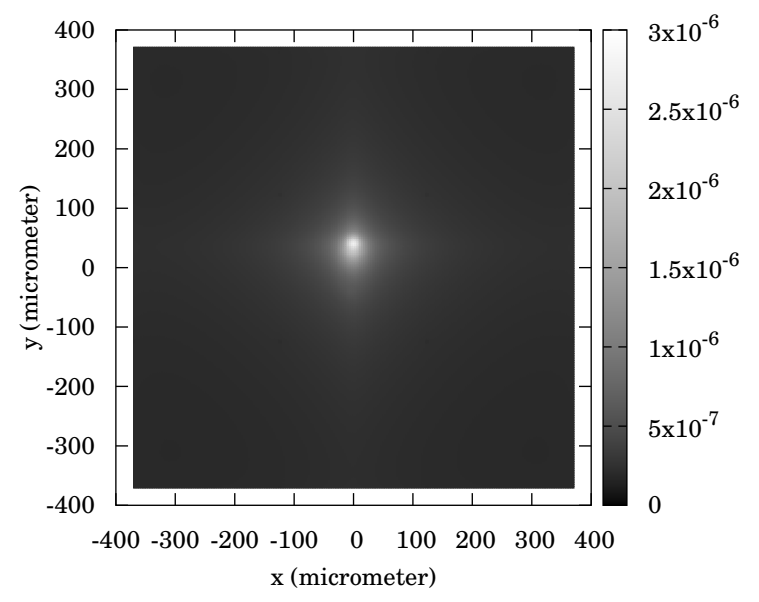

(a)

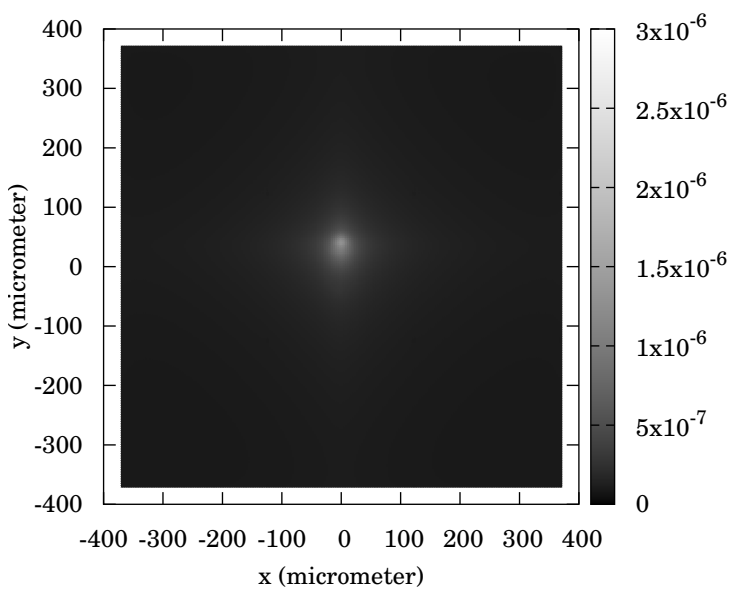

(b)

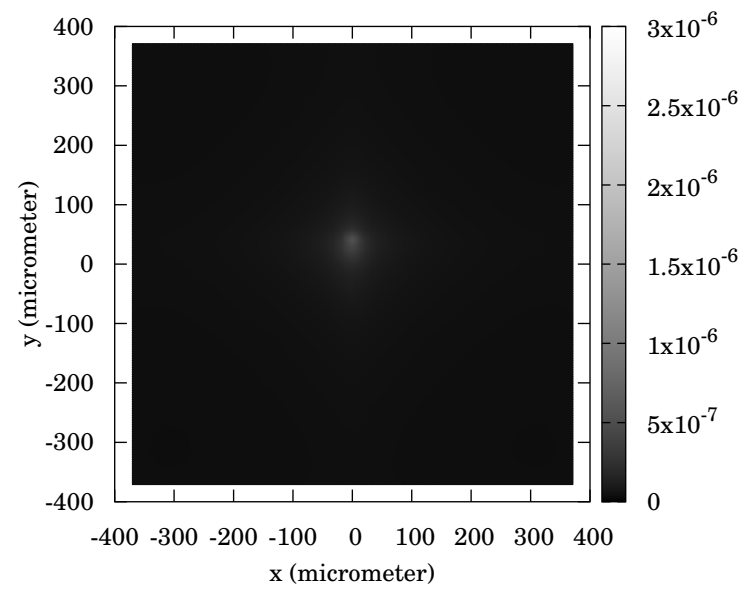

(c)

Figure 6: The focus intensity distributions for a particle with $a=2.0 \mu \mathrm{m}, b=1.0 \mu \mathrm{m}, \alpha_{\mathrm{p}}=45^{\circ}$, and $\beta_{\mathrm{p}}=90^{\circ}$. The incident angle is $\beta_{\mathrm{s}}=62^{\circ}$, and the effective distance between the particle and the plane surface is $d=0.1 \mu \mathrm{m}(\mathrm{a})$, $d=0.5 \mu \mathrm{m}(\mathrm{b})$, and $d=1.0 \mu \mathrm{m}(\mathrm{c})$. 


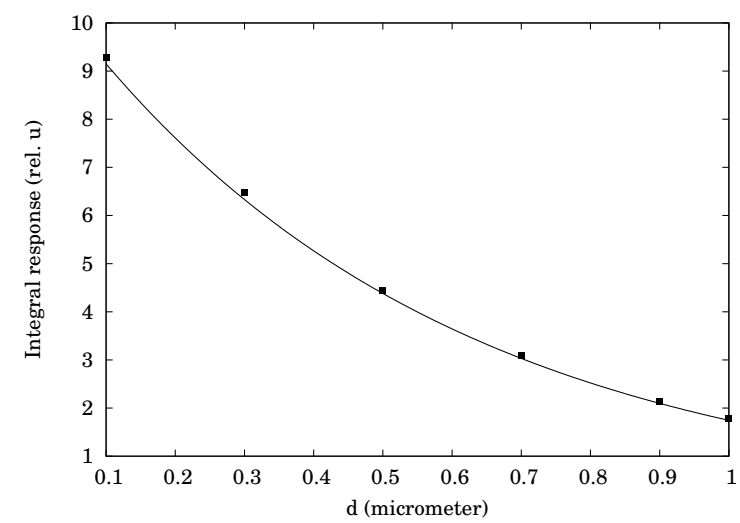

Figure 7: Integral response of the same particle as in Fig. 6 as a function of the effective distance between the particle and the plane surface $d$. The solid curve is the exponential function fitted to the data (dots).

[4] Michael A. Bevan and Dennis C. Prieve. Direct measurement of retarded van der waals attraction. Langmuir, 15(23):7925-7936, nov 1999.

[5] Laurent Helden, Kilian Dietrich, and Clemens Bechinger. Interactions of colloidal particles and droplets with water-oil interfaces measured by total internal reflection microscopy. Langmuir, 32(51):13752-13758, dec 2016.

[6] Jeffrey A. Fagan, Paul J. Sides, and Dennis C. Prieve. Evidence of multiple electrohydrodynamic forces acting on a colloidal particle near an electrode due to an alternating current electric field. Langmuir, 21(5):1784-1794, mar 2005 .

[7] Christopher L. Wirth, Paul J. Sides, and Dennis C. Prieve. The imaging ammeter. Journal of Colloid and Interface Science, 357(1):1-12, may 2011.

[8] Michael A. Bevan and Dennis C. Prieve. Forces and hydrodynamic interactions between polystyrene surfaces with adsorbed PEO-PPO-PEO. Langmuir, 16(24):9274-9281, nov 2000.

[9] Daniel J. Beltran-Villegas, Tara D. Edwards, and Michael A. Bevan. Self-consistent colloidal energy and diffusivity landscapes in macromolecular solutions. Langmuir, 29(40):12337-12341, sep 2013.

[10] Dzina Kleshchanok, Remco Tuinier, and Peter R. Lang. Depletion interaction mediated by a polydisperse polymer studied with total internal reflection microscopy. Langmuir, 22(22):9121-9128, oct 2006.

[11] Simon Biggs, Raymond R. Dagastine, and Dennis C. Prieve. Oscillatory packing and depletion of polyelectrolyte molecules at an oxide-water interface. The Journal of Physical Chemistry B, 106(44):11557-11564, nov 2002.

[12] Stacy G Bike. Measuring colloidal forces using evanescent wave scattering. Current Opinion in Colloid 8 Interface Science, 5(1-2):144-150, mar 2000.

[13] Michael A. Bevan and Shannon L. Eichmann. Optical microscopy measurements of kT-scale colloidal interactions. Current Opinion in Colloid \& Interface Science, 16(2):149-157, apr 2011.

[14] Laurent Helden, Elena Eremina, Norbert Riefler, Christopher Hertlein, Clemens Bechinger, Yuri Eremin, and Thomas Wriedt. Single-particle evanescent light scattering simulations for total internal reflection microscopy. Applied Optics, 45(28):7299, oct 2006.

[15] Christopher Hertlein, Norbert Riefler, Elena Eremina, Thomas Wriedt, Yuri Eremin, Laurent Helden, and Clemens Bechinger. Experimental verification of an exact evanescent light scattering model for TIRM. Langmuir, 24(1):1-4, jan 2008.

[16] Seymour Stein. Addition theorem for spherical wave functions. Quarterly of Applied Mathematics, 19(1):15-24, 1961.

[17] Adrian Doicu, Thomas Wriedt, and Yuri A. Eremin. Light Scattering by Systems of Particles. Springer Berlin Heidelberg, 2006. 
[18] Marcel Leutenegger, Ramachandra Rao, Rainer A. Leitgeb, and Theo Lasser. Fast focus field calculations. Optics Express, 14(23):11277-11291, 2006.

[19] Clayton T. McKee, Spencer C. Clark, John Y. Walz, and William A. Ducker. Relationship between scattered intensity and separation for particles in an evanescent field. Langmuir, 21(13):5783-5789, jun 2005.

[20] Aidin Rashidi and Christopher L. Wirth. Motion of a janus particle very near a wall. The Journal of Chemical Physics, 147(22):224906, dec 2017. 